\section{HALLUCINOGENIC PERSISTING PERCEPTION DISORDER: A CASE SERIES AND REVIEW OF THE LITERATURE}

'Hannah HF Ford, ${ }^{2}$ Clare CF Fraser, 'Emma ES Solly, ${ }^{1,3}$ Joanne JF Fielding, ${ }^{3,4}$ Owen OW White, ${ }^{4,5,6}$ Anneke AWWan der Walt. ${ }^{1}$ Department of Neurosciences, The Alfred Hospital, Melbourne, VIC, Australia; ${ }^{2}$ Neuro-Ophthalmology, Sydney University, Sydney, NSW, Victoria; ${ }^{3}$ Ocular Motor Research Laboratory, Monash University, Clayton, VIC, Australia; ${ }^{4}$ Neuro-ophthalmology Service, The Alfred Hospital, Melbourne, VIC, Australia; ${ }^{5}$ Multiple Sclerosis and Neuroimmunology (MSNI), The Alfred Hospital, Melbourne, VIC, Australia; ${ }^{6}$ Multiple Sclerosis and Neuro-ophthalmology Group, Monash University, Clayton, VIC, Australia

\subsection{6/bmjno-2021-ANZAN.79}

Objectives To report the clinical characteristics and investigation findings of a series of Hallucinogenic Persisting Perception Disorder (HPPD) cases and review previous HPPD case reports from the literature.

Methods Case studies were collected from consultant neuroophthalmologists between 2019 and 2020. PubMed and MEDLINE databases were searched for case reports between 2000 and 2020 using the terms 'hallucinogenic persisting perception disorder' and 'case report'

Results Thirteen case studies were reviewed. Lysergic acid diethylamide (LSD), 3,4-Methyl enedioxy methamphetamine (MDMA) and cannabinoids were the most common drugs used prior to HPPD onset. Twenty-two different visual symptoms were described. The most commonly reported were visual snow, floaters, palinopsia, photophobia and photopsia. Ophthalmic and neurologic investigations were normal. Two patients fully recovered after benzodiazepine treatment or no treatment. Twenty-four literature case reports were identified. LSD, MDMA and cannabinoids were the most frequent drugs used. Seventeen different visual symptoms were described. Ophthalmic and neurologic investigations showed no clinically significant findings in the majority of cases. 25\% of cases fully recovered after treatment with benzodiazepines, eye movement desensitisation and reprocessing therapy, anti-epileptic drugs or no treatment.

Conclusions A wide variety of hallucinogenic and non-hallucinogenic recreational substances are implicated in HPPD. Clinical presentation includes a diverse range of positive visual phenomena and overlaps with Visual Snow Syndrome (VSS). Neurologic and ophthalmic investigations are typically normal. Management is complicated due to a lack of high-quality evidence. Controlled trials are needed to better understand the pathophysiology and optimize treatment for HPPD.

\section{THE DIAGNOSTIC JOURNEY OF MITOCHONDRIAL DISEASE PATIENTS}

\footnotetext{
1,2Laura | Rudaks, ${ }^{1}$ Eloise Watson, 'Michal Lubomski, ${ }^{1}$ Fabienne Edema-Hildebrand, ${ }^{1}$ Kate Ahmad, ${ }^{1}$ Christina Liang, ${ }^{1,3}$ Ryan Davis, ${ }^{1,3}$ Carolyn Sue. ${ }^{1}$ Royal North Shore Hospital, St Leonards, NSW, Australia; ${ }^{2}$ Neurology Unit, Concord Repatriation General Hospital, Concord, NSW, Australia; ${ }^{3}$ Kolling Institute, St Leonards, NSW, Australia
}

\subsection{6/bmino-2021-ANZAN.80}

Introduction Mitochondrial disorders can often be challenging to diagnose and patients may undergo protracted investigative odysseys before reaching a diagnosis. ${ }^{1}$ This study reviewed the diagnostic journey for genetically confirmed Mitochondrial Disease patients.

Methods Patients with a genetic diagnosis of mitochondrial disease seen at the Department of Neurogenetics, Royal
North Shore Hospital, were invited to complete an online survey at their appointment or via telephone. Participant clinical records were reviewed for additional data, including genetic diagnosis.

Results Between October 2018 and April 2020, survey results were obtained from 68 patients. The most common presenting symptoms were fatigue (39\%), weakness (31\%), and droopy eyelids (31\%). The most frequently completed investigations were MRI (55\%), neurophysiologic testing (45\%) and EEG (44\%). $33 \%$ of participants had consulted five or more doctors with an overall mean time to diagnosis of 6.2 years. $41 \%$ of patients received a diagnosis within two years of symptom onset, $31 \%$ between 5 and 15 years, and 11\% after 15 years or more. $38 \%$ of participants received at least one alternative diagnosis prior to their definitive genetic mitochondrial disease diagnosis. Following diagnosis, 34\% of patients joined a support group and $87 \%$ felt that this was beneficial.

Conclusions Our results demonstrate that many patients experience long delays, undergo many investigations and see multiple doctors before a diagnosis of mitochondrial disease is reached. It is hoped that advances in diagnostic pathways and access to earlier genetic testing may streamline the process. ${ }^{2}$

\section{REFERENCES}

1. Grier J, Hirano M, Karaa A, et al. Diagnostic odyssey of patients with mitochondrial disease. Results of a survey. Neurol Genet 2018;4: e230. doi:10.1212 NXG.0000000000000230

2. Watson E, Davis R, Sue C. New diagnostic pathways for mitochondrial disease. J Trans/ Genet Genom 2020;4:188-202

\section{IGLON5 AUTOIMMUNITY IN TWO CASES WITH PERIPHERAL NERVOUS SYSTEM FEATURES}

1,2 Laura I Rudaks, ${ }^{3,4}$ Victor SC Fung, ${ }^{5}$ Jean-Pierre Halpern, ${ }^{2,5}$ Omar Ahmad. 'Concord Repatriation General Hospital, Concord, NSW, Australia; ${ }^{2}$ Royal North Shore Hospital, St Leonards, NSW, Australia; ${ }^{3}$ Neurology Department, Westmead Hospital, Westmead, NSW, Australia; ${ }^{4}$ Sydney Medical School, University of Sydney, Sydney, NSW, Australia; ${ }^{5}$ Neurology Department, Sydney Adventist Hospital, Wahroonga, NSW, Australia

\subsection{6/bmjno-2021-ANZAN.8}

Introduction IgLON5 autoimmunity has four main clinical patterns; a sleep disorder, bulbar syndrome, PSP-like pattern and predominant cognitive impairment. Other manifestations include movement disorders, gait instability, dysautonomia and neuropsychiatric features [1-3]. Peripheral nervous system involvement has been occasionally reported $[3,4]$. We describe two cases of IgLON5 autoimmunity presenting with peripheral neuropathy.

Cases A 72-year-old lady presented with progressive distal lower limb numbness, paraesthesia, incoordination and gait disturbance. Associated features included fluctuating facial numbness, limb and trunk fasciculations, upper limb tremor, anxiety, episodic hyperventilation, nocturnal myoclonus and vocalisations in sleep. Nerve conduction studies (NCS) demonstrated demyelinating features in the lower limbs. AntiIgLON5 antibodies were detected in cerebrospinal fluid. She was treated with IVIg, oral prednisolone, azathioprine and plasma exchange.

A 73-year-old man presented with worsening tremor. Evolving features included facial paraesthesia, imbalance, head 'fogginess', visual agnosia, constipation, insomnia, sleep utterances, somnambulism, nocturnal tremor and myoclonus. NCS showed a generalised demyelinating sensorimotor polyneuropathy. Neuropathy screen demonstrated anti-IgLON5 antibodies and IgG 